tischer Leukämie erhielt, zeigte Reifungsdefekt des Knochenmarkes. Urinextrakte zweier normaler Personen ergaben keine spezifischen Zellwucherungen. Die angegebene Harnextraktionsmethode ist eine Modifikation von MoCullaghs Methode zur Extraktion von Androgen aus Urin. Werner Schultz (Charlottenburg-Westend). ${ }^{\circ}$

Beck, Franees F., and John C. Krantz jr.: Glyeolysis in tumor tissue. 3. The effect of ultrasonic vibrations on the growth and glycolysis of Walker sareoma 319. (Glyko1yse im Tumorgewebe. 3. Die Wirkung von Ultraschallwellen auf Wachstum und Glykolyse des Walker-Sarkoms 319.). (Dep. of Pharmacol., School of Med., Univ. of Maryland, Baltimore.) Amer. J. Canc. 39, 245-250 (1940).

Ultraschallwellen mit einer Frequenz von 300000 Schwingungen pro Sekunde dringen durch Haut und Unterhautbindegewebe bis in ein subcutan gesetztes WalkerSarkom 319 durch. Solide Tumoren adsorbieren hierbei stärker als überflüssigte die Energie, was infolge ihrer Umsetzung in Wärme durch thermoelektrische Messungen ermittelt wurde. Wiederholte Behandlung mit Ultraschallwellen soll den Milchsäuregehalt der Tumoren (Bestimmung nach Friedemann und Gräser) schwach erhöhen und das Tumorwachstum in geringem Maße herabsetzen. (2. vgl. diese Z. 47, 461.)

\title{
Hormone, Vitamine.
} A. Rosenbohm (Hamburg).

Rodewald, W.: Innere Sekretion und Krebsbereitsehait. (Biol. Abt., Inst. gegen Geschwulstkrankh., Rudolf Virchow-Krankenh., Berlin.) Gesdh.führ. H. 6, 210-217 (1941).

Untersuchungen an Tieren haben gezeigt, daß bei mit Krebs belasteten Tieren schon im frühen Alter eine verminderte Aktivität der Hormondrüsen vorliegt. Beim carcinomkranken Menschen ist hierüber nichts Sicheres bekannt. Doch haben Untersuchungen ergeben, daß bei Frauen mit späterem Gebärmutterhalsearcinom die erste Regelblutung erst mit 16-17 Jahren einsetzt, die Blutungen verfrüht aufhören. In Gegenden, in denen der Kropf gehäuft auftritt, soll auch die Zahl der Krebserkrankungen erhöht sein. Gesunde Verwandte Krebskranker in kropfarmen Gegenden wiesen einen erniedrigten Grundumsatz und Neigung zu Alkalose auf, der Blutzucker fand sich erhöht, es bestand deutliche Überempfindlichkeit gegen Kohlehydrate. Bei Krebskranken und deren Blutsverwandten fand sich häufig ein erniedrigter Blutkalkspiegelwert. Die Befunde bedürfen der Ergänzung, um festzustellen, ob sich in einer Unter funktion der Drüsen mit innerer Sekretion tatsächlich eine Krebsbereitschaft äußern kann.

Annelise Schlüter (Göttingen).

Geist, Samuel H., and Udall J. Salmon: Are estrogens eareinogenic in the human female? The effect of long-continued estrogen administration upon the uterine and vaginal mucosa of the human female. (Kann man mit Follikelhormon bei der Frau Krebs erzeugen?) (Gynecol. Serv., Mt. Sinai Hosp., New York.) Amer. J. Obstetr. 41, $29-36$ (1941).

Monatelang fortgesetzte Behandlung mit Follikelhormon führte bei Frauen in der Menopause zu geordnetem Aufbau des Scheidenepithels, im Endometrium höchstens zu glandulärer Hyperplasie. Nach Aussetzen der Behandlung nahmen die Schleimhäute wieder das vorherige Bild der Atrophie an. - Nach 1-1,5 Millionen i. B.E. kam es meist zu einer Blutung; das Endometrium befand sich dann nicht immer im Zustand der glandulären Hyperplasie. - Die größte verabreichte Dosis betrug 53,4 Millionen i. B.E. in 38 Monaten.

Büttwer (Bonn).。

\section{Metastasen.}

Kogan, A.: L'influenee du choe sur la formation des metastases dans le cancer clinique. (Einfluß des Shocks auf die Bildung von Metastasen in Fällen von klinischem Krebs.) Akuš, i Ginek. Nr 5, 13-17 (1941) [Russisch].

Der Autor erwähnt am Anfang seiner Abhandlung seine Experimente mit $478 \mathrm{Ka}$ ninchen, welchen der Brown-Pearce-Tumor in die Genitalien inokuliert war und 